\title{
Fluorescence detection of bladder cancer using urine cytology
}

\author{
CHIT YAW FU ${ }^{1}$, BENG KOON NG ${ }^{1}$, SIRAJUDEEN GULAM RAZUL ${ }^{1}$, WILLIAM WEI LIM CHIN ${ }^{2}$, \\ PUAY HOON TAN ${ }^{3}$, WEBER KO LAU ${ }^{4}$ and MALINI OLIVO ${ }^{2}$ \\ ${ }^{1}$ School of Electrical and Electronic Engineering, Nanyang Technological University; \\ ${ }^{2}$ Division of Medical Sciences, National Cancer Centre; Departments of ${ }^{3}$ Pathology \\ and ${ }^{4}$ Urology, Singapore General Hospital, Singapore
}

Received February 15, 2007; Accepted April 23, 2007

\begin{abstract}
Bladder cancer is the fourth most common malignant disease worldwide, accounting for $4 \%$ of all cancer cases. In Singapore, it is the ninth most common form of cancer. The high mortality rate in bladder cancer can be reduced by early treatment following pre-cancerous screening. Currently, the gold standard for screening bladder tumors is histological examination of biopsy specimens, which is both invasive and time-consuming. In this study, ex vivo urine fluorescence cytology was investigated to offer an alternative timely and biopsy-free means for detecting bladder cancers. Sediments in patient urine samples were extracted and incubated with a novel photosensitizer, hypericin. Laser confocal microscopy was used to capture the fluorescence images at an excitation wavelength of $488 \mathrm{~nm}$. Images were subsequently processed to single out the exfoliated bladder cancer cells from the other cells based on the cellular size. Intensity histograms of each targeted cell and feature vectors, derived from the histogram moments, were used to represent each sample. A difference in the distribution of the feature vectors of normal and low-grade cancerous bladder cancer cells were observed. A diagnostic algorithm for discriminating between normal and low-grade cancerous cells is elucidated in this report. This study suggests that the fluorescence intensity profiles of hypericin in bladder cells can potentially provide an automated quantitative means of early bladder cancer diagnosis.
\end{abstract}

\section{Introduction}

Bladder cancer is the fourth most common form of malignant disease worldwide, accounting for $4 \%$ of all cancer cases $(1,2)$. In Singapore, bladder cancer is the ninth most common

Correspondence to: Dr Malini Olivo, Division of Medical Sciences, National Cancer Centre, 11 Hospital Drive, Singapore 169610 E-mail: dmsmcd@nccs.com.sg

Key words: bladder cancer, fluorescence imaging, photodynamic diagnosis, hypericin, image analysis form of cancer (3). Mortality rates are very high due to invasive bladder cancer $(4,5)$. The high mortality rate has therefore initiated active research in early detection, reliable diagnosis and effective treatment of bladder cancer. White-light cystoscopy combined with random biopsy has been the gold standard for screening bladder tumors. However, the process of examination is time-consuming and is associated with patient discomfort. Flat lesions such as dyplasia and carcinoma in situ (CIS) are frequently overlooked due to the poor contrast given by white-light illumination (6). Thus, a non-invasive and timely technique for detecting early cancer is highly desirable.

Urine cytology is a non-invasive screening technique that analyses the exfoliated bladder cells from voided urine. Conventional urine cytology is suitably used for the detection of CIS, but not for early or low-grade bladder cancer (7). A few studies have suggested that cancer cells can be identified with application of a photosensitizer as a selective marker. The unique photo-activated properties of photosensitizers have been studied and applied for both photodynamic therapy (PDT) $(8,9)$ and photodynamic diagnosis (PDD) of cancer (10). Fluorescence cytology (FC) using aminolevulinic acid (ALA) was reported as a new diagnostic modality for the detection and follow-up of bladder cancer (11). FC combines the advantages of PDD and urine cytology to allow the noninvasive detection of bladder cancer with high sensitivity and specificity. A disadvantage of this technique in the past has been that patients were required to receive intravesical instillation of photosensitizer. Previous findings from our group have demonstrated a biopsy-free screening technique that synergizes PDD and ex vivo fluorescence cytology without the need for intravesical instillation (12).

In PDD, using hypericin, a potent photosensitizer derived from Hypericum perforatum is used to enhance the fluorescence contrast between normal and tumor cells. A number of in vitro and in vivo studies have also reported the use of hypericin as a selective tumor marker for bladder cancer with no cytotoxic effects (13-16).

Previous elementary analysis on ex vivo fluorescence cytology has suggested the diagnostic potential in detecting early bladder cancer. In this work, advanced image processing techniques were used to quantify the fluorescence properties of urine sediments for cancer diagnosis. Automated screening was implemented to single out bladder cancer cells based 
on fluorescence intensity analysis. The fluorescence intensity histograms were examined and correlated to the differentiated cellular uptake of hypericin between normal and bladder tumor cells. A set of concise descriptors was derived from the histogram to represent the various bladder pathology. Finally, a Bayes classifier was adopted to discriminate between benign and low-grade tumor cells. The diagnostic performance of the developed algorithm was compared with that of the cytological examination. The preliminary results obtained show promise and suggest that low-grade bladder cancer cells may be identified with a high degree of accuracy using this methodology.

\section{Materials and methods}

Sample set. In this study, 110 fluorescence microscopic images of urine sample were taken from 21 patients at the Singapore General Hospital. Histological examinations were also performed on the urine sample of each patient and they were classified as normal, low-grade cancer, high-grade cancer or inflammatory histologies. This study was reviewed and approved by the Ethics Committee of the Singapore General Hospital and National Cancer Centre Singapore.

Hypericin preparation. Hypericin from Molecular Probes was used and a stock solution was dissolved in dimethyl sulphoxide (DMSO). Serum-free tissue culture medium RPMI-1640 supplemented with $1 \mathrm{X}$ L-glutamine, 100 units/ml penicillin and streptomycin (Gibco) and 1X sodium pyruvate was used as the incubation medium.

Urine cytology preparation. Urine samples were collected from patients who underwent bladder cancer screening. Samples were processed for fluorescence cytology immediately after specimen collection. Voided urine was first centrifuged to a pellet at 3,000 rpm for $15 \mathrm{~min}$ at $18^{\circ} \mathrm{C}$. Supernatant was then removed and the pellet was re-suspended with $1 \mathrm{ml}$ of hypericin-containing culture medium. The cell suspension was incubated in the dark for $15 \mathrm{~min}$ at room temperature. The cells were then centrifuged again at 3,000 rpm for $15 \mathrm{~min}$ at $18^{\circ} \mathrm{C}$. Subsequently, the hypericin solution was removed and the sediment was rinsed with phosphate-buffered saline (PBS). Similar centrifugation was done to separate the cells from the hypericin residues. After decanting the supernatant, the remaining cell pellet was smeared onto a microscopic slide and covered with a cover slip.

Fluorescence microscopic imaging. A confocal fluorescence microscope (Carl Zeiss, LSM 510) was used to capture fluorescence images of samples excited by a $488 \mathrm{~nm}$ wavelength Argon laser. Within each specimen, up to 7 microscopic fields were randomly sampled. An objective lens (Plan Neofluor 10x, 0.3 NA) was chosen to effectively collect the fluorescence signal with sufficient spatial resolution for image analysis. An optical filter was used to detect hypericin-induced fluorescence emission between the wavelength of $590 \mathrm{~nm}$ and $700 \mathrm{~nm}$. The fluorescence images were recorded with a dimension of $512 \times 512$ pixels in an 8-bit format, yielding a resolution of approximately $1.8 \mu \mathrm{m}$ per pixel.

Image processing. Image processing of the collected fluo- rescence data were performed using Matlab ${ }^{\circledR}$ (The MathWorks, Inc., Natick, MA). The fluorescence images were first preprocessed to single out bladder cells for image analysis. Elliptical bladder cells were individually selected based on the length of the minor and major axes. To minimize computational time, background noise and red blood cells were first eliminated from the images. Background noise was removed by setting the lower and upper intensity thresholds at 5 and $95 \%$ of the maximum intensity level respectively. A standard morphological filtering was applied to get rid of objects with dimensions $<6 \mu \mathrm{m}$. This technique allows the red blood cells to be detected and removed from the images. Intensity histograms were then extracted from the processed images and the corresponding first three moments were calculated. The moments of the histograms were used as a set of concise descriptors to represent the bladder cell images of each patient. The nth moment of a normalized histogram about its mean was calculated using:

$$
\mu_{n}(I)=\sum_{k=1}^{N}\left(I_{k}-m\right)^{n} f\left(I_{k}\right)
$$

where

$$
m=\sum_{k=1}^{N} I_{k} f\left(I_{k}\right)
$$

is the mean of the intensity $I, N$ is the total number of pixels and $f\left(I_{\mathrm{k}}\right)$ is the probability density function of the normalized histogram at intensity value $I_{\mathrm{k}}$.

Bayes classification technique was used to develop discrimination rules based on the derived moments of the intensity histograms. The idea is to derive a decision function $d$ using Bayes theorem which minimizes the probability of classification error. An unknown feature vector $x$, derived from histogram moments, is labeled as class $\omega_{i}$ if $d_{i}(x)>d_{j}(x)$ for all $j \neq i$. Assuming that the moment vector $x$ is normally distributed, the resultant decision function is simplified to (17):

$$
\left.d_{j}(x)=\ln P\left(\omega_{j}\right)-\frac{1}{2} \ln \left|C_{j}\right|-\frac{1}{2}\left[\left(x-m_{j}\right)^{T} C_{j}^{-1}\left(x-m_{j}\right)\right] \text { iii }\right)
$$

where $P\left(\omega_{j}\right)$ is the prior probability of occurrence of class $\omega_{j}$ and is assumed to be equal for every class. The mean vector $m_{j}$ and covariance matrix $C_{j}$ are defined as:

$$
m_{j}=\frac{1}{N_{j}} \sum_{x \in \omega_{j}} x
$$

and

$$
C_{j}=\frac{1}{N_{j}}\left(\sum_{x \in \omega_{j}} x x^{T}\right)-m_{j} m_{j}^{T}
$$

respectively, where $N_{j}$ is the number of moment vectors from class $\omega_{j}$.

\section{Results}

Image preprocessing. Microscopic images of treated specimen were captured for investigating the differentiated cellular 

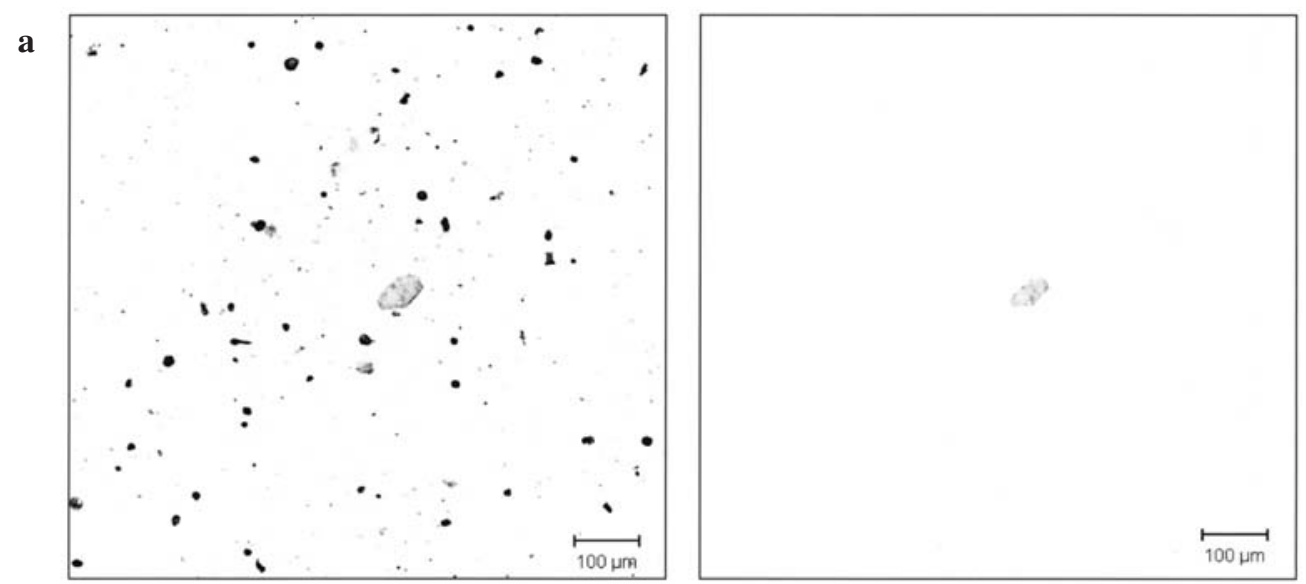

Figure 1. (a) Original and (b) processed fluorescence image from a normal specimen. Negative images are presented for clear illustration.

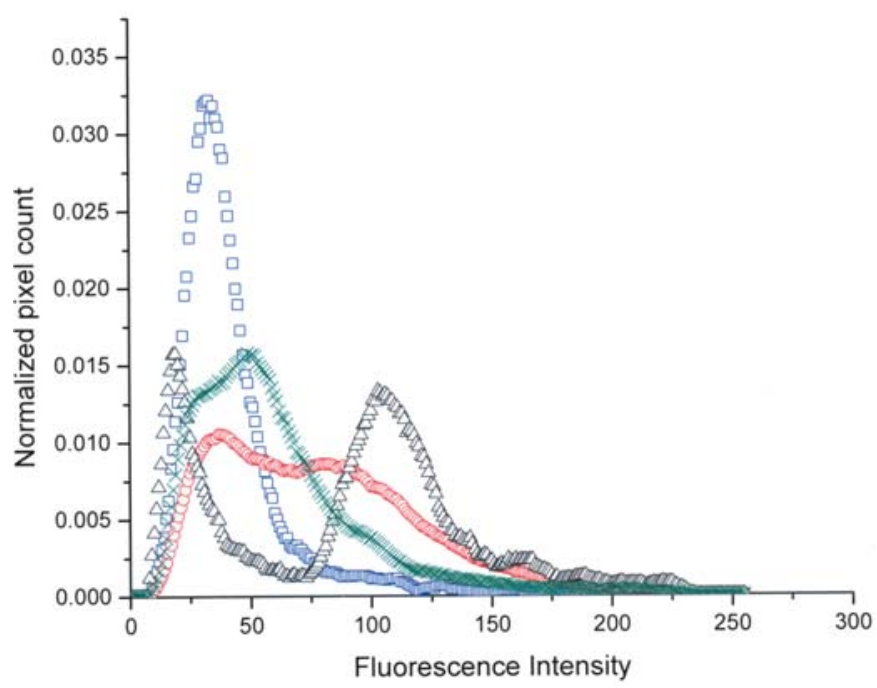

Figure 2. Typical histogram data from the processed fluorescence images of normal $(\square)$, low-grade tumor $(\circ)$, high-grade tumor $(\Delta)$ and inflammatory (x) bladder classes.

uptake of hypericin in normal and cancerous bladder cells. Urine cytology studies reveal that several components, such as exfoliated urothelial cells, red blood cells, crystalline contaminants, are commonly observed in urinary sediments (18). In our experiments, all these components were found to take up hypericin as well, thereby complicating the microscopic fluorescence analysis. Since red blood cells have a typical diameter of between 5 and $8 \mu \mathrm{m}$, red blood cells could be effectively removed using image dilation without the exclusion of the larger bladder cells, which measure between 20 and $50 \mu \mathrm{m}$ in diameter (18). In addition, intensity thresholding was implemented to reject pixels corresponding to background noise in the image.

Transitional or urothelial cell linings on the wall of bladder are of particular interest because $>90 \%$ of all bladder cancers are transitional cell carcinomas (TCC) (7). In this work, a direct image processing technique was implemented to single out urothelial cell from the image using its inherent cellular parameters as the selection criteria. Since biological cells are generally elliptical, major axis length and minor axis length were used to characterize the physical structure of an urothelial cell. A number of histological samples for urine cytology were examined to quantify the axis lengths of cells. The average axis lengths of urothelial cells were estimated to be between 20 and $80 \mu \mathrm{m}$. Urothelial cells can originate from either the superficial or the inner layer of the bladder mucosa. The superficial cells are generally larger than the inner cells. Due to the limited image resolution, the smaller cells typically gave a smaller pixel area. Consequently the inner urothelial cells were excluded from the intensity histogram analysis. Selection rules were also implemented to extract superficial urothelial cells from the images. The major axis length is set between 40 and $80 \mu \mathrm{m}$, while the limit of the minor axis length is set between 20 to $80 \mu \mathrm{m}$.

In addition, superficial urothelial cells from the same urine sample can also vary in size. Smaller cellular areas were observed when the cells were folded or shrunk due to osmosis. Furthermore, multiple cells can also clump together and the intensity information of each cell cannot be easily separated. These overlapping cells were also omitted from the analysis in this work.

It was also observed that the targeted bladder cells exhibit an abrupt increase in the fluorescence intensity at its periphery with thickness of about 3 to $5 \mu \mathrm{m}$. These high intensity regions at the cell periphery were also excluded from the histogram analysis. Fig. 1a illustrates a fluorescence image captured from a normal specimen. The processed image in Fig. 1b shows the target cell after eliminating background noise and irrelevant components.

Intensity histogram. Fig. 2 shows the representative histogram data from the processed fluorescence images. The data were collected from four classes of patients, i.e. patients diagnosed with normal bladder, low-grade urothelial tumor, high-grade urothelial tumor and inflammation. Intensity histogram was obtained for each image with the exclusion of background pixels. Histograms of the specimens belonging to the same class were then summed and normalized into a single histogram. The resulting histogram represents the mean statistical distribution of the fluorescence intensity of each class. In the present study, specimens which have less than three urothelial cells were omitted in the analysis. This 

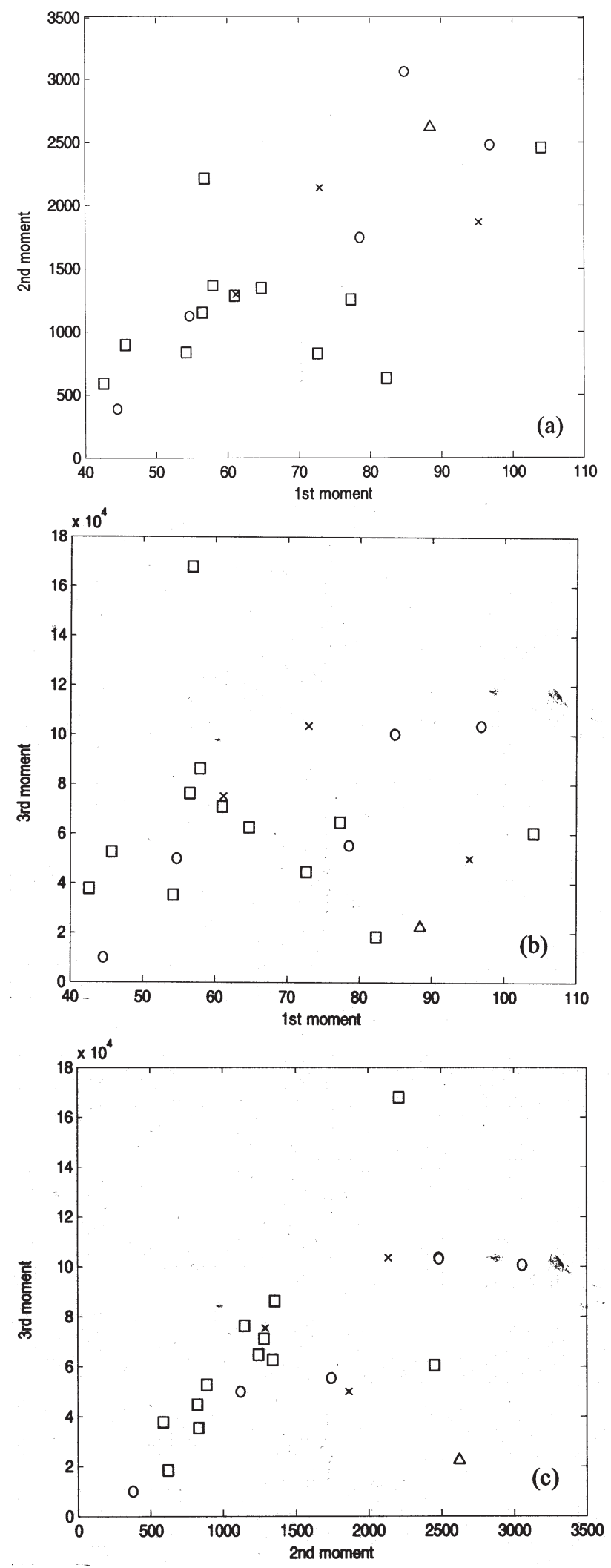

Figure 3. 2-D scatter plots of moments from normal ( $\square$ ), low-grade tumor $(\circ)$, high-grade tumor $(\Delta)$ and inflammatory $(\mathrm{x})$ classes.

is because the number of cell pixels is too small to exhibit a normally distributed histogram. As can be seen from Fig. 2, each class exhibits distinct features in the mean histogram profile. Feature descriptors were extracted from the histogram of individual patient as described in Materials and
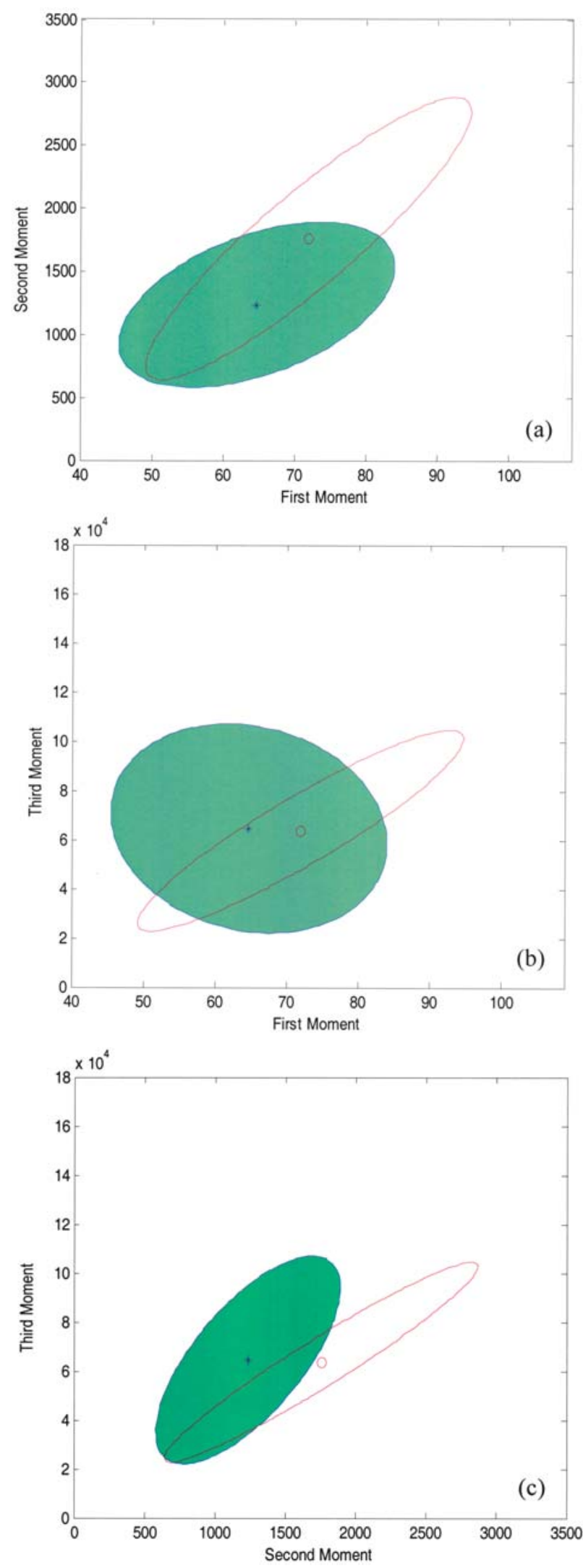

Figure 4. 2-D plots showing the computed moment distributions of normal (filled) and low-grade tumor (open) classes. The outline of each region defines the FWHM of the normal distribution and the means are denoted by symbols $(*, \circ)$.

methods under 'Image processing' and were used to develop the algorithm for classifying the cell groups. 
Table I. Level of sensitivity and specificity in differentiating benign tissue and low-grade tumor based on urine cytology and the diagnostic algorithm developed in this study.

\begin{tabular}{lcc}
\hline Classification & Sensitivity $\%$ & Specificity $\%$ \\
\hline Urine cytology & 87 & 40 \\
Bayes classifier & 93 & 100 \\
\hline
\end{tabular}

Discrimination algorithm. The shape of each histogram was described using its moments. It is obvious from Fig. 2 that there are variations in the shape of the histogram profiles among the four different classes of patients. In this analysis, the first three moments are adopted to interpret the key features of a histogram. The first moment describes the mean fluorescence intensity, the second moment measures the spread of the intensity histogram about the mean intensity and the third moment quantifies its symmetry with respect to the mean intensity. Fig. 3 shows the two-dimensional plot of the histogram moments of the 21 samples.

The moments of the histograms of normal bladder and low-grade tumor were fitted to a normal distribution. The data of high-grade tumor and inflammatory bladder classes were excluded in the fitting due to its limited dataset. The fitted normal distributions of the moments of normal bladder and low-grade tumor classes are plotted in Fig. 4. The moment distributions of these two classes are highly overlapping, since both cancerous and normal bladder cells can be found in the urine specimen of cancer patients. The computed mean intensity and second moment of the low-grade tumor class were found to be higher as compared to that of normal class. These results indicate that the cells from the low-grade tumor class tend to emit a stronger fluorescence with greater variance. On the other hand, the third moment of normal class is just slightly above that of low-grade class. The minor increment is attributed to its lower mean intensity and the lower intensity threshold value implemented in image processing. Consequently, the histogram appears to skew to the right and yields a larger positive value for its third moment.

Discrimination of the pattern classes using the decision function based on Bayes classifier was carried out on the sample set. The level of sensitivity and specificity achieved for Bayes classification between the benign (normal and inflammatory) and low-grade tumor classes are tabulated in Table I. In addition, the performance of urine cytology is also compared with that of the Bayes classifier. The comparison reveals that the use of the Bayes classifier has improved the diagnostic performance as opposed to conventional urine cytology.

\section{Discussion}

There is a pronounced contrast between normal class and low-grade tumor class, as illustrated by the histogram analysis (Fig. 2). The peak of the intensity profile in the normal class closely matches the first intensity peak of the low-grade tumor class. The second peak of the low-grade tumor occurs at a higher intensity value of about 80 . This observation suggests that there are two groups of urothelial cells emitting at different intensity levels. Since the fluorescence intensity originates from hypericin, the observed intensity peaks can be correlated to the differentiated cellular uptake of hypericin in cancerous specimens.

There is a growing body of evidence showing the superior ability of hypericin in detecting bladder cancer based on in vivo and in vitro studies. Colorimetric parameters, which are extracted from in vivo fluorescence images, are also successfully used to quantify the relative amount of hypericin in tissue for cancer diagnosis (16). The outcome of these studies has substantiated a preferential uptake of hypericin by tumor cells, giving rise to a stronger fluorescence emission as compared to that of normal cells. Hence, the histogram data obtained in this work is in good agreement with the other reported findings. As expected, the inclusion of fluorescence signals from tumor cells causes an increase in the mean intensity and a broadening of the histogram profile. In addition, the degree of slant is also slightly reduced. These three key features can be used to discriminate between benign and tumor cells. It is apparent that this technique does not induce any patient discomfort during specimen collection. A prompt computational diagnosis can be carried out 1-h after chemical treatment of urine sediments with hypericin. Hence, this enables an immediate medical attention to the patient following the detection of early bladder cancer.

The extraction of the diagnostic information is largely limited by three factors. First, undesirable cell conditions can complicate the image analysis. The current processing approach is incapable of resolving the intensity information of clumped and folded cells. Besides, shrunken cell are also inappropriate for analysis due to their limited pixel numbers. Secondly, the limited cell yield in the urine specimen is a crucial issue to perform fluorescence urine cytology. This is also the theoretical limit with respect to conventional cytologic detection of urinary bladder neoplasm. Neither morphologic criteria nor ancillary techniques will detect a concurrent urothelial neoplasm if the urine fails to sample cancerous cells or cellular constituents (19). The efficacy of urine cytology can be substantially improved if a non-invasive medical treatment is available to increase the cell yields in urine specimen for the purpose of bladder diagnosis. The third factor relates to the number of red blood cells present in the urine samples, which can sometimes dominate the urinary sediment and may completely obscure the identification of the other cells. Rapid advances in microfluidics and MEMS technology may help to circumvent this problem in the future.

This study has not addressed the possibility of improvement on the diagnostic contrast by studying the optimal incubation time of urinary sediments in hypericin and the time-dependent intensity of specimens after drug incubation. The optimal cellular localization of hypericin in in vitro studies was reported to be 2 to $4 \mathrm{~h}$ post drug incubation (15). However, it is questionable if the viability of exfoliated urothelial cells is still retained for proper interaction with hypericin after extended immersion in urine. Consequently, there exists a compromise between the localization time and degree of cell viability. In order to ensure cell viability, bladder cells were observed under fluorescence microscope 
within $1 \mathrm{~h}$ of chemical treatment. Besides, the fluorescence images show that there is an abrupt increase in fluorescence intensity at the vicinity of the cell membrane. This bright region could be attributed to the accumulation of hypericin molecules incorporated in the phospholipid bilayers of the cell membrane prior to complete drug localization in specific cell organelles. In this study, the optimized observation time point has yet to be determined.

In conclusion, fluorescence image analysis was investigated to supplement the PDD of bladder cancer based on ex vivo urine cytology. In this preliminary study, an attempt was made to correlate the fluorescence intensity profile of microscopic images with the histopathological state of bladder cells. The images were first preprocessed to single out superficial urothelial cells for analysis on intensity histograms. The diagnostic information was successfully retrieved after digitally filtering out the background noise and irrelevant constituents. Characteristic features of the histogram profiles were observed for four distinct classes of bladder conditions. In particular, a stronger second fluorescence intensity peak was detected from the cancerous specimens, suggesting a preferential localization of hypericin in the cancerous cells. The first three moments were extracted from each histogram to quantify the histogram shapes and the Bayes technique was carried out to classify targeted cells based on the derived moments. It was shown that the discrimination between benign cells and low-grade tumor cells using the corresponding feature vectors is very promising. Furthermore, its diagnostic performance is found to surpass that of conventional urine cytology. This study indicates that the fluorescence intensity profile of hypericin in bladder cells can potentially be used in the early diagnosis of bladder cancer.

\section{Acknowledgements}

The authors wish to thank National Medical Research Council of Singapore and National Cancer Centre for their funding and facilities and Ms. Vanaja Manivasager for editorial assistance.

\section{References}

1. D'Hallewin M, Bezdetnaya L and Guillemin F: Fluorescence detection of bladder bancer: a review. Eur Urol 42: 417-425, 2002.

2. Kamat A and Lamm D: Chemoprevention of urological cancer. J Urol 161: 1748-1760, 1999.
3. Chia K, Seow A, Lee H and Shanmugaratnam K: Cancer Incidence in Singapore 1993-1997. Singapore Cancer Registry, 2000.

4. Bray F, Sankila R, Ferlay J and Parkin D: Estimates of cancer incidence and mortality in Europe in 1995. Eur J Cancer 38: 99-166, 995.

5. Black RJ, Bray F, Ferlay J and Parkin DM: Cancer incidence and mortality in the European Union: Cancer Registry dates and estimates of national incidence for 1990. Eur J Cancer 33: 1075-1077, 1990.

6. Kriegmair M, Zaak D, Rothenberger KH, Rassweiler J, Jochanm D, Eisenberger F, Tauber R, Stenzl A and Hofstetter A: Transurethral resection for bladder cancer using 5-aminolevulinic acid induced fluorescence endoscopy versus white light endoscopy. J Urol 168: 475-478, 2002.

7. Han M and Schoenberg MP: The use of molecular diagnostics in bladder cancer. Urol Oncol 5: 87-92, 2000.

8. Dolmans DE, Fukumura D and Jain R: Photodynamic therapy for cancer. Nat Rev Cancer 3: 380-387, 2003.

9. Hugh B, Catherine K, Janelle R and Nicolas S: Clinical aspects of photodynamics therapy. Sci Prog 85: 131-150, 2002.

10. Loschenov VB, Konov VI and Prokhorov AM: Photodynamic Therapy and Fluorescence Diagnostics. Laser Physics 10: 1188$1207,2000$.

11. Pytel A and Schmeller N: New aspect of photodynamic diagnosis of bladder tumors: fluorescence cytology. Urology 59: 216-219, 2002.

12. Olivo M, Lau W, Manivasager V, Bhuvaneswari R, Wei Z, Soo KC, Cheng C and Tan PH: Novel photodynamic diagnosis of bladder cancer: ex vivo fluorescence cytology using hypericin. Int J Oncol 23: 1501-1504, 2002.

13. D'Hallewin MA, Kamuhabwa A, Roskams T, Witte AD and Baert L: Hypericin-based fluorescence diagnosis of bladder carcinoma. Br J Urol 89: 760-763, 2002.

14. Kamuhabwa AA, Roskams T, Baert L and De Witte PA: Microscopic quantification of hypericin fluorescence in an orthotopic rat bladder tumor model after intravescial instillation. Int J Oncol 22: 933-937, 2003.

15. Olivo M, Lau W, Manivasager V, Tan PH, Soo KC and Cheng C: Macro-microscopic fluorescence of human bladder cancer using hypericin fluorescence cystocopy and laser confocal microscopy. Int J Oncol 23: 983-990, 2002.

16. Kah JCY, Olivo M, Lau WKO and Sheppard CJR: Pathological diagnosis of bladder cancer by image analysis of hypericin induced fluorescence cystoscopic images. Proc SPIE-OSA Biomed Optics, 5863, 2005.

17. Gonzalez RC and Woods RE: Digital Image Processing. Prentice-Hall, New Jersey, 2002.

18. Koss LG: Diagnostic Cytology of the Urinary Tract. LippincottRaven, New York, 1996.

19. Bastacky S, Ibrahim S, Wilczynski SP and Murphy WM: The accuracy of urinary cytology in daily practice. Cancer $87: 118$ $128,1999$. 